\title{
Die Fleischfarbene Fingerwurz (Dactylorhiza incarnata), Orchidee des Jahres 2015
}

\author{
Kurt Baumann $\dagger$
}

\begin{abstract}
Early marsh-orchid (Dactylorhiza incarnata) is orchid of the year 2015. Its morphology, biology, distribution, endangering and variability are shown.
\end{abstract}

\section{Zusammenfassung}

Die Fleischfarbene Fingerwurz (Dactylorhiza incarnata) ist Orchidee des Jahres 2015. Ihre Morphologie, Biologie, Verbreitung, Gefährdung und Variabilität werden vorgestellt.

\section{Einordnung und Name}

Die zum 27. Mal gewählte Orchidee des Jahres gehört zu einer Gattung, die in Mitteleuropa nicht nur bezüglich der Anzahl der Sippen (Arten und Unterarten), sondern auch bei manchen Arten nach der Individuenzahl eine der größten ist. Die Arbeitskreise Heimischer Orchideen (AHO) wählten 1989 mit der Breitblättrigen Fingerwurz (Dactylorhiza majalis) die erste Orchidee des Jahres. Im Jahr 2008 folgte als zweite Art der Gattung die Übersehene Fingerwurz (Dactylorhiza praetermissa) und nun die Fleischfarbene Fingerwurz oder das Fleischfarbene Knabenkraut, wie die Pflanze vor nicht langer Zeit noch genannt wurde.

Bereits die sogenannten Väter der Botanik wie Brunfels (1532) und Fuchs (1543) unterschieden bei den einheimischen Orchideen solche mit glatten, runden Knollen und andere mit handförmig geteilten. Wegen ihrer Blütezeit Anfang Mai (in der Kreuzwoche) wurden Letztere im Mainzer und Frankfurter Raum umgangssprachlich als Creutzblum bezeichnet.

Früher waren die heute zu Dactylorhiza gestellten Orchideen mit der Gattung Orchis (Knabenkraut) vereinigt. Die Auftrennung der Gattung Orchis in eine Gruppe mit glatten Knollen (Orchis) und in eine zweite Gruppe mit handförmig geteilten Knollen (Dactylorhiza) nahmen Botaniker im Jahr 1947 vor. Die deutsche Übersetzung Fingerwurz wurde von Sun-

Abb. 1: Fleischfarbene Fingerwurz in der Übersicht.

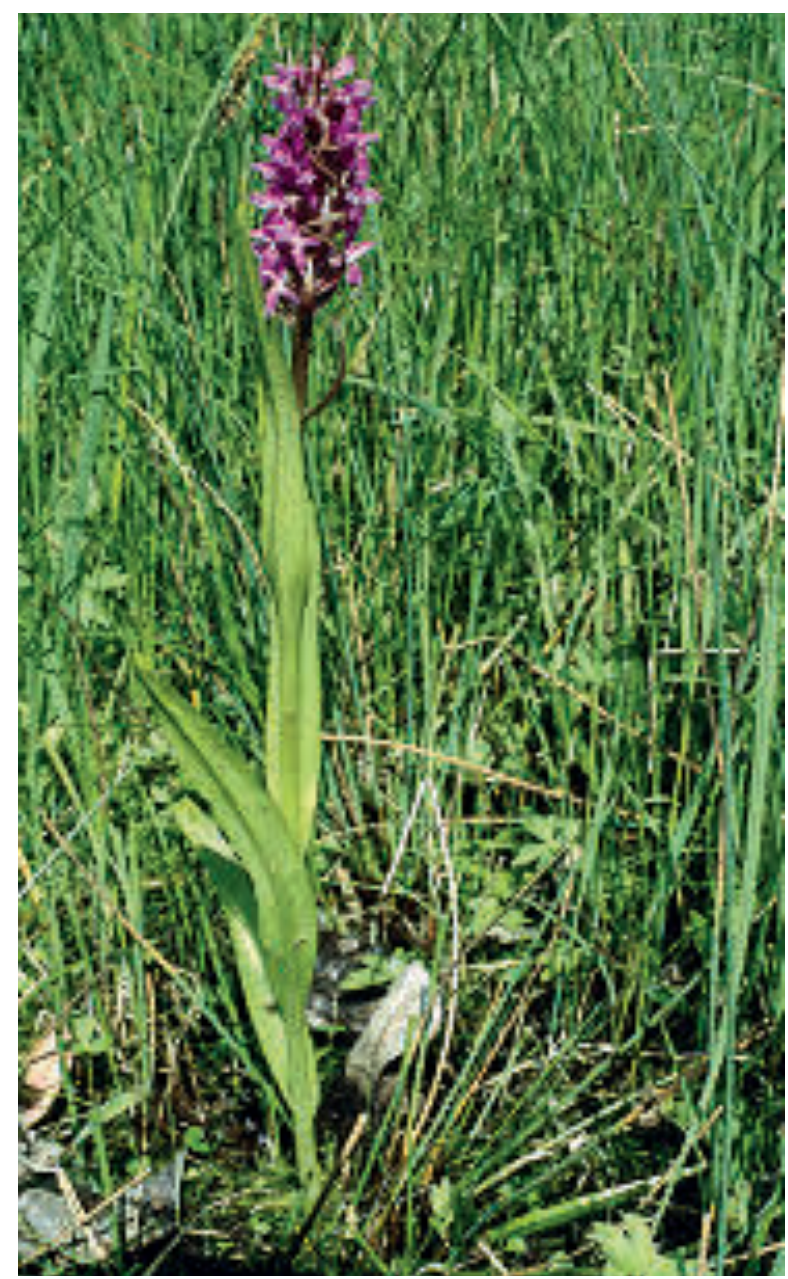

DERMANN vorgeschlagen und hat sich inzwischen durchgesetzt (AHO 2005).

Die beiden Gattungen sind nicht nur durch ihre Wurzelknollen zu unterscheiden. Die echten Knabenkräuter haben häutige Tragblätter, die kürzer als der Fruchtknoten sind, eine Blattrosette und nur 1-3 scheidige Stängelblätter. Außerdem verfügen sie über einen 2-3-teiligen 


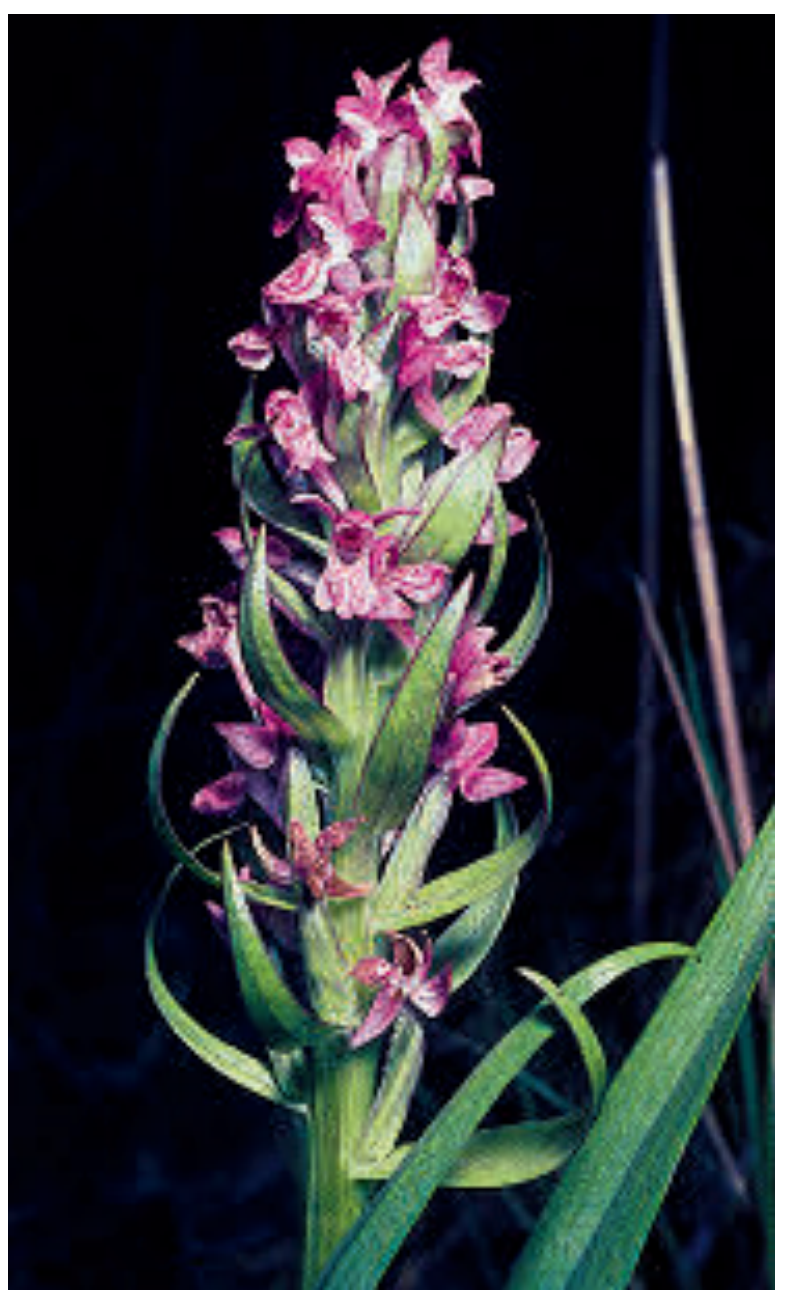

Lippen-Mittellappen. Bei den Arten der Gattung Fingerwurz sind mindestens die untersten Tragblätter laubblattartig (krautig mit Quernerven) und länger als der Fruchtknoten. Der Stängel ist mehrblättrig, und der Lippen-Mittellappen ist meist ungeteilt (JäGER 2011).

Der deutsche Name Fleischfarbene Fingerwurz ist eine Übersetzung des wissenschaftlichen Artnamens (lat. incarnatus = zu Fleisch geworden). Nach dem ungültigen Synonym $D$. strictifolia wird sie auch als Steifblättrige Fingerwurz bezeichnet. Der Name Kuckucksblume, der auch für Orchideen aus anderen Gattungen verwendet wird (u .a. Platanthera) sollte wegen seiner Uneindeutigkeit nicht benutzt werden. Weitere regionale Volksnamen sind Feldzinkli, Jungfernblume und Katzenschweif. Die Bezeichnung Engelshand bezieht sich auf die geteilten Knollen (FüLler 1962).

Die Gliederung der Gattung Dactylorhiza ist strittig. Nach molekulargenetischen Untersuchungen und Enzymanalysen werden Dactylorhiza incarnata und D. fuchsii mit einer Chromosomenzahl von $2 \mathrm{n}=40$ als reinerbige Arten angesehen. Aus diesen beiden sind vermutlich durch Hybridisierung und Polyploidisierung weitere Dactylorhiza-Arten hervorgegangen (Ausnahme: D. sambucina). Einige werden heute deshalb manchmal nur noch als Unterarten von D. majalis angeführt (Kretzschmar 2008). Dementsprechend unterscheiden sich die Angaben zur Artenzahl von Dactylorhiza je nach Autor nicht unerheblich voneinander. Nach AHO (2005) gehören rund 50 Arten und Unterarten zur Gattung Dactylorhiza, nach Baumann et al. (2006) sind es 60 Arten und Unterarten, hinzu kommen 10 Varietäten. JäGER (2011) nennt sogar 75 Arten. In Deutschland kommen nach JäGER (2011) 12 Dactylorhiza-Arten vor, nach Kretzschmar (2008) dagegen nur 5. Letzterer stellt die Hohlzunge (Coeloglossum viride) als Dactylorhiza viridis ebenfalls zur Fingerwurz.

\section{Bau der Pflanze}

Zur Blütezeit hat $D$. incarnata zwei unterirdische Wurzelknollen. Die blühende Pflanze ist aus der vorjährigen Knolle hervorgegangen, die dann abstirbt. Zuvor ist in der Achsel des untersten schuppenartigen Niederblattes eine Tochterknolle entstanden. Im Herbst entwickelt sich aus der Knolle ein Sprossabschnitt bis zur Erdoberfläche, der im Frühjahr weiterwächst. Die Knollen sind 2-3 cm lang, 2,5-3 cm breit und in 2-4 lange, wurzelähnliche Fortsätze tief handförmig geteilt. Normale Nebenwurzeln befinden sich am Knollenansatz.

Die Pflanze wird normalerweise $20-60 \mathrm{~cm}$ hoch, ausnahmsweise auch bis $80 \mathrm{~cm}$. Ihr kräftiger Stängel ragt steif aufrecht und ist ziemlich dick ( $1 \mathrm{~cm}$ und mehr), aber hohl, und vor allem oberwärts kantig. Seine hellgrüne Farbe kann im oberen Teil purpurn überlaufen sein.

Der Stängel ist hoch hinauf beblättert. An

Abb. 2 (oben): Blütenstand von Dactylorrhiza incarnata.

Abb. 3 (Seite 61): Ausschnitt aus dem Blütenstand. 
ihm sind 3-8 grüne, ungefleckte Blätter mehr oder weniger gleichmäßig, wechselständig verteilt (Presser 2002). Sie sind schmal lanzettlich, gekielt bis gefaltet und stehen steif aufrecht. Sie sind 13-31 cm lang (nach ReInHARdT 1991 nur bis $20 \mathrm{~cm}$ ), $1-3 \mathrm{~cm}$ breit und lang zugespitzt. Die Spitze ist bei den oberen Blättern kapuzenförmig zusammengezogen.

\section{Blüte und Bestäubung}

Die Blütezeit reicht von Mai bis Anfang Juli. Der Blütenstand, der im Knospenzustand nicht von einem Blatt umschlossen wird, ist eine 6-12 cm lange Ähre. Die krautigen Tragblätter, in deren Achseln die einzelnen Blüten sitzen, sind mit bis $27 \mathrm{~mm}$ Länge relativ groß, rein grün bis purpurn überlaufen und lanzettlich zugespitzt. Der Blütenstand besteht aus 10-60 (meist 15-40) hell- bis dunkelrosa (fleischfarbenen) Blüten. Selten sind sie ganz weiß.

Die Blüte sitzt auf dem 9-13 mm langen, um 180 Grad gedrehten, grünlichen Fruchtknoten. Die zwei seitlichen, äußeren Sepalen sind 6-9 mm lang, eiförmig und nach hinten oben geschlagen. Das kleinere mittlere äußere Sepalum bildet mit den beiden seitlichen, schief eiförmigen Blütenblättern einen geschlossenen Helm, der das Säulchen mit den Fortpflanzungsorganen schützt.

Die rhombische Lippe ist durch die Drehung des Fruchtknotens nach unten gerichtet. Sie ist 5-6 $\mathrm{mm}$ lang und 6-9 $\mathrm{mm}$ breit, schwach dreilappig und leicht gefaltet. Ihre Seitenlappen sind abwärts gebogen, der Mittellappen ist vorgezogen. Das Zentrum der Lippe ist heller gefärbt mit einem dunkelroten, einfachen oder doppelten Schleifenmuster. Der Sporn ist etwas kürzer als der Fruchtknoten, konisch, stumpf und waagerecht bis leicht abwärts gebogen (Künkele \& Baumann 1998). Er enthält keinen Nektar. Dementsprechend ist die Fleischfarbene Fingerwurz wie auch die anderen Orchideen der Gattungen Orchis und Dactylorhiza eine Nektartäuschblume. Dennoch zeigt die unregelmäßige Verteilung der Früchte am Fruchtstand bei einem Fruchtansatz von 70-83\% bzw. 67\% das Vorliegen von Fremdbestäubung durch Insekten (AHO 2005).

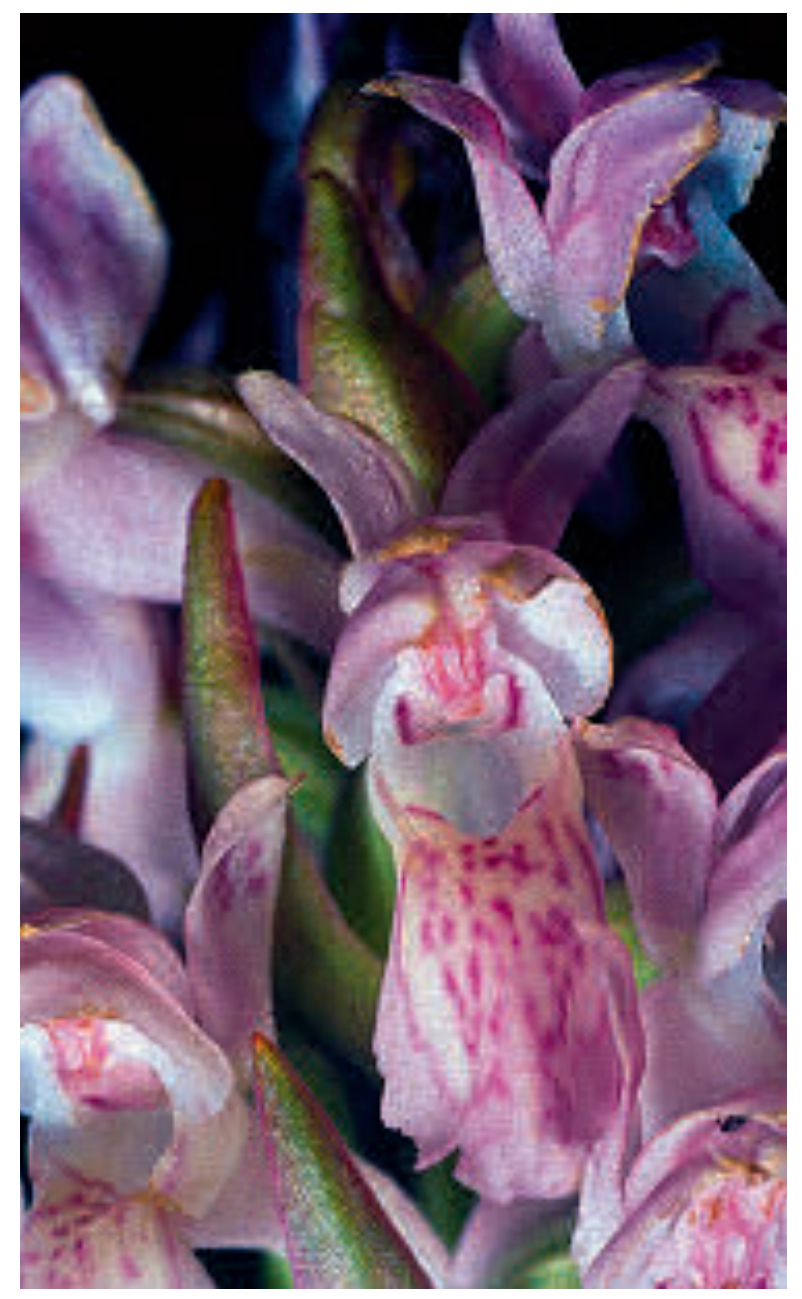

Über dem Sporneingang befindet sich das Säulchen (Gynostemium), entstanden durch Verwachsung des kurzen Griffels und der Narbe mit dem einzigen Staubblatt. Auf der nach unten gerichteten Seite, gegenüber dem Sporneingang, trägt das Säulchen die stark klebrige Narbe. Über der Narbe befindet sich ein kurzer Fortsatz des Säulchens (Rostellum), an dessen Vorderseite das Staubblatt befestigt ist. Der Pollen ist durch klebrige Fäden zu einem keulenförmigen Paket zusammengefasst (Pollinium). Die Pollinien sind lang gestielt und enden jede in einer Klebscheibe (FüLLER 1962).

Als Bestäuber kommen vorwiegend Hummeln und solitäre Bienen in Frage. Wenn sie sich auf die Lippe setzen und mit dem Rüssel den (nicht vorhandenen) Nektar im Sporn zu erreichen versuchen, kommen sie mit ihrem Kopf zwangsläufig in die Nähe der Pollinien, die sich mit Hilfe der Klebscheiben am Kopf 


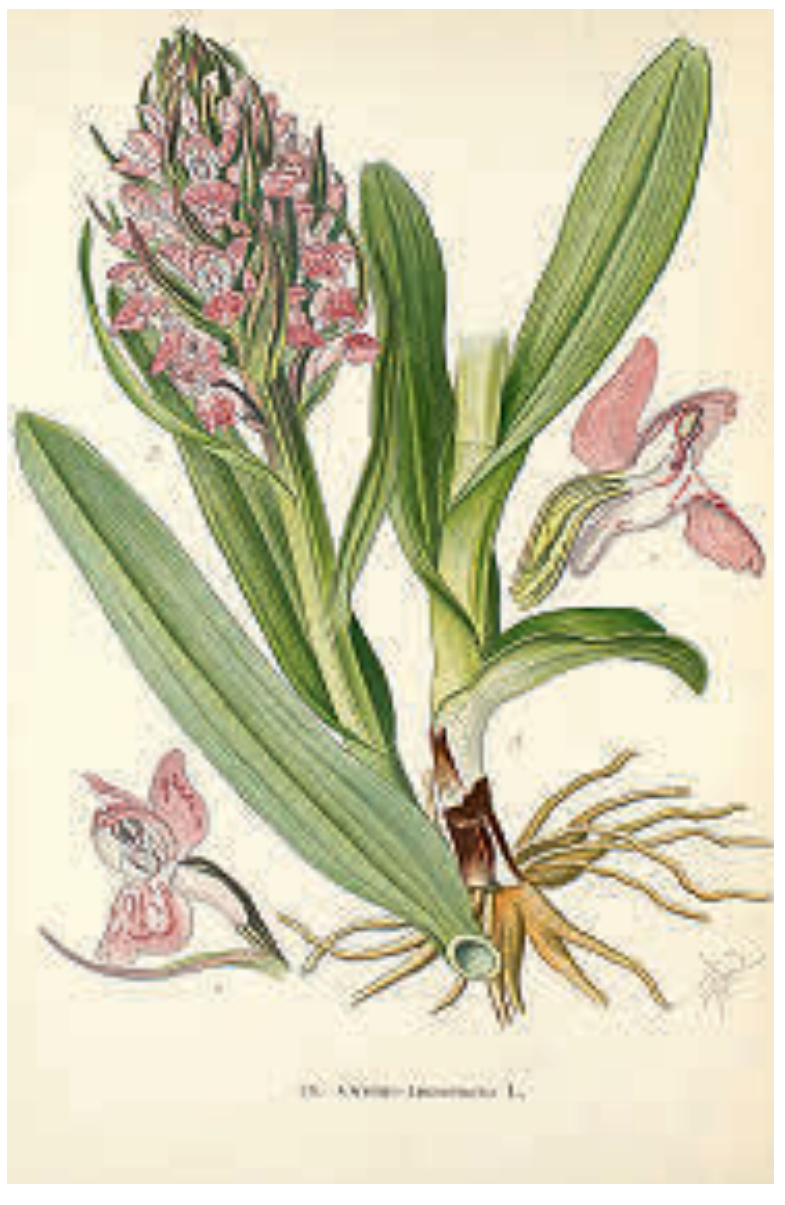

oder Rüssel des Insekts festheften. Die Stielchen stehen zuerst aufrecht, knicken dann im rechten Winkel nach vorn ein. Beim Besuch der nächsten Blüte kommen die Pollenpakete, die nun ungefähr parallel zum Rüssel stehen, mit der Narbe in Berührung und bleiben dort kleben (FüLLER 1962).

Ein Pollinium von Dactylorhiza incarnata enthält nach AHO (2005) 48600 Pollenkörner. Diese können dann nach einem einmaligen Bestäubungsvorgang für die Befruchtung der zahlreichen Samenanlagen im Fruchtknoten sorgen. Die Samen enthalten keine Nährstoffe und sind sehr klein (0,5-0,8 mm lang, 0,16-0,2 mm breit, AHO 2005). Die Kapselfrüchte sind ab Mitte August reif, 9-16 mm lang und 5-7 mm breit. Die Samen sind von einer blasenförmigen Hülle aus großen, verkorkten Zellen umschlossen. Sie werden bei Erschütterung freigesetzt und vom Wind ausgebreitet.

Wegen der fehlenden Nährstoffe ist die Orchidee in der Anfangsphase ihrer Entwicklung von einem Pilz abhängig. Die in die Zellen des Keimlings eingedrungenen Pilzfäden werden abgetötet, verdaut und liefern die für die Weiterentwicklung notwendigen Stoffe. Nach der Ausbildung des ersten grünen Blattes ist die Orchidee vom Pilz unabhängig (Selosse 2007).

\section{4. Ökologie und Verbreitung}

Die Fleischfarbene Fingerwurz ist eine Lichtpflanze, mäßig wärmebedürftig und ein Nässezeiger. Sie wächst auf stickstoffarmen, mageren, schwach sauren bis leicht basischen Standorten. Sie gedeiht in Sümpfen und an Quellflüssen, in Feucht- und Binsenwiesen, in nährstoffarmen Mooren, im Flussschotter sowie in Seggen- und Schilfbeständen. Bei lückigem Bewuchs ist sie auch in Bruchwäldern mit jungen Erlen und Weiden und in Pfeifengras-Streuwiesen anzutreffen. Gegenüber der Breitblättrigen Fingerwurz (Dactylorhiza majalis) bevorzugt sie magerere Böden. Die typische Fleischfarbene Fingerwurz kommt in Deutschland bis 1400 m Höhe vor.

Dactylorhiza incarnata ist vor allem durch Nutzungsumwandlung von Grünland durch Aufforstung, Düngung und Trockenlegung gefährdet. Nach AHO (2005) liegt ein Rückgang von $56,37 \%$ vor, was für einheimische Orchideen im mittleren Bereich liegt. Für ihren Erhalt ist der Schutz extensiv bewirtschafteter Feuchtwiesen mit Pufferzonen zur Vermeidung von Eutrophierung wichtig.

Die Fleischfarbene Fingerwurz ist eine eurasiatisch verbreitete Art. Sie kommt in fast ganz Europa von der submeridionalen bis zur borealen Zone vor. Als Nordgrenze wird die Finnmark angegeben, als Südgrenze die Gebirge Südspaniens, Mittelitalien und Nordgriechenland. Auch in der Nordtürkei und auf der Krim kommt sie vor. Ihre Verbreitung reicht vom Atlantik bis fast zum Pazifik, wobei die Ostgrenze in Ostsibirien (Jakutien, Dahurien) nicht klar ist (BAUMANN 2006).

In Deutschland liegen die Hauptvorkommen im Alpenvorland, entlang des Oberrheins

Abb. 4 (oben): Zeichnung von Dactylorhiza incarnata, ganze Pflanze und Blüten im Detail. 
und im nordostdeutschen Tiefland (Mecklenburg-Vorpommern, Brandenburg). Sonst ist sie vor allem entlang der großen Flusssysteme verbreitet.

\section{Variabilität}

Wie bei anderen Orchideenarten kommt es bei der Fleischfarbenen Fingerwurz zu Variationen in den Größenverhältnissen, der Färbung und anderen Merkmalen. Dies führt zu Beschreibungen von Sippen, über deren Bewertung die Autoren verschiedener Meinung sind.

In den Streuwiesen des Oberrheins und auf Usedom kommt eine frühblühende, hellrosa Form vor, die mit der dunkleren, fleischfarbenen Normalform vergesellschaftet ist. Nur selten bilden sie Übergangsformen. Grund dafür sind vermutlich unterschiedliche Bestäuber. In Oberschwaben tritt eine ebenfalls frühblühende, weiße Varietät hyphaematodes auf. Sie unterscheidet sich von der Normalform durch gefleckte Blätter. Manche Botaniker differenzieren noch die Varietät haematodes, deren deutlich abgesetzte Punkte nur auf der Blattoberseite vorhanden sind, während hyphaematodes Flecken auf Ober- und Unterseite zeigt. Eine kleinwüchsige Varietät ist serotina, die auch später blüht (Ende Juni bis Anfang Juli). Ihre Verbreitung ist unklar. Vermutlich kommt sie heute nur noch im Alpenvorland vor. Deutlich abgegrenzt und deshalb von manchen als eigene Art und nicht nur als Unterart angesehen ist die Sippe ochroleuca mit gelblich-weißen Blüten. Sie blüht 1-2 Wochen später als die Normalform und kommt in Deutschland in BadenWürttemberg und Bayern im Alpenvorland vor. Nicht für Deutschland nachgewiesen ist die hochalpine Sippe cruenta, die ab 1000 m Höhe in den Alpen (Südostfrankreich bis Westösterreich) in Skandinavien und im Baltikum vorkommt. Sie wird bisweilen als eigene Art be- wertet und hat meist gefleckte Blätter, einen niedrigeren Wuchs und dunklere Blüten.

Weitere für Europa beschriebene Sippen sind die Varietäten lobelii (großblütig, in den Niederlanden) und pulchella (purpurviolette Blüten, in Großbritannien). Dazu kommen die Unterarten baumgartneria (endemisch in Aserbeidschan) und coccinea mit niedrigem Wuchs und scharlachrotfarbenen Blüten, die in nassen Dünensenken, aber auch im Binnenland (in Schottland und Wales) gedeiht.

Zudem wurden Hybriden mit anderen europäischen Arten der Gattung Dactylorhiza nachgewiesen. Bei der Variationsbreite der einzelnen Arten ist die Zuordnung der Hybriden allerdings nicht immer ganz einfach oder eindeutig. Interessanterweise ist auch ein Bastard mit der Hohlzunge (Coeloglossum viride), die heute zur Gattung Dactylorhiza gestellt wird, bekannt. Auch ein Gattungsbastard mit der Händelwurz (Gymnadenia conopsea) wurde beobachtet. Wie Dactylorhiza hat die Händelwurz händchenförmig geteilte Wurzelknollen.

\section{Literatur}

Arbeitskreise Heimischer Orchideen (Hrsg.) 2005: Die Orchideen Deutschlands. - Uhlstädt-Kirchhasel. Baumann, H., Künkele, S. \& Lorenz, R. 2006: Die Orchideen Europas. - Stuttgart.

Füller, F. 1962: Die Gattungen Orchis und Dactylorchis. Die Orchideen Deutschlands, 3. Teil. Neue. - Wittenberg. JäGER, E.-J. (Hrsg.) 2011: Rothmaler. Exkursionsflora von Deutschland. Grundband. 20. Aufl. - Heidelberg.

Kretzschmar, H. 2008: Die Orchideen Deutschlands und angrenzender Länder. - Wiebelsheim.

Künkele, S., Baumann, H. 1998: Orchidaceae. In: Sebald,O., Philippi,G. \& Seybold, S.: Die Farn- und Blütenpflanzen Baden-Württembergs. - Bd. 8. Stuttgart. Presser, H. 2002: Orchideen. 2. Aufl. - Hamburg. Reinhardt, H. R. et al. 1991: Die Orchideen der Schweiz und angrenzender Gebiete. - Egg.

Selosse, M. \& Guillaumin, J. 2007: Vom Keimstadium zur adulten Pflanze: Die symbiontischen Pilze der Orchideen. - Jahresber. Naturwiss. Ver. Wuppertal 60: 253-273. 